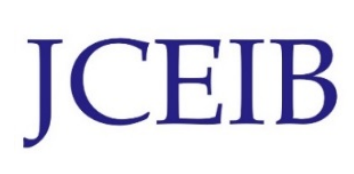

Journal Chemical Engineering and Industrial Biotechnology (JCEIB) Open Access

Volume 2 pp. 1-12; September 2017

CUniversiti Malaysia Pahang Publisher

DOI: https://doi.org/10.15282/JCEIB-V1-02.29/9/2017/2.2

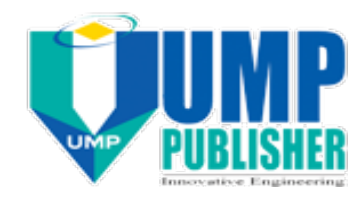

\title{
BIOSYNTHESIS OF SILVER NANOPARTICLES USING MARINE MICROALGAE ISOCHRYSIS sp.
}

\author{
Tevan, R ${ }^{\mathrm{a}, *}$, Saravanan Jayakumar ${ }^{\mathrm{a}}$, Nor Haledah Ahmad Sahimi ${ }^{\mathrm{a}}$, Nur Farah Ain Iqbal ${ }^{\mathrm{a}}$, \\ Iffah Zapri ${ }^{\mathrm{a}}$, Natanamurugaraj Govindan ${ }^{\mathrm{a}}$, Mohd Hasbi Ab. Rahim ${ }^{\mathrm{a}}$, Solachuddin J.A. \\ Ichwan $^{\mathrm{b}}$, Gaanty Pragas Maniam ${ }^{\mathrm{a}}$ \\ ${ }^{a}$ Bioprocess Laboratory, Faculty of Industrial Sciences \& Technology, Universiti Malaysia \\ Pahang, Lebuhraya Tun Razak, 26300 Gambang, Kuantan, Pahang, Malaysia \\ ${ }^{\mathrm{b}}$ Kulliyyah of Dentistry, International Islamic University Malaysia, Bandar Indera Mahkota, \\ 25200 Kuantan, Pahang, Malaysia \\ ${ }^{*}$ Corresponding author: tevandrs@gmail.com \\ Tel.: +609-5492915 Fax.: +609-5492888
}

\begin{abstract}
Biosynthesis of metal nanoparticles has received a remarkable attention due to their eco-friendly and potential applications in pharmaceutical and medical fields. The searches for natural alternatives to replace biosynthetic nanoparticles have resulted in extensive studies of microalgal derived metal nanoparticles. Since there are very limited reports on Isochrysis sp. in synthesising metal nanoparticles, a novel initiative was taken to induce an environmentally friendly and low cost technique to biosynthesise the silver nanoparticles (AgNPs) using marine microalgae, Isochrysis sp. Further, the synthesised silver nanoparticles were screened against human pathogen for antimicrobial effects. The characterisation of nanoparticles were confirmed by UVvisible spectroscopy, field emission scanning electron microscopy (FESEM), Fourier transform infrared spectroscopy (FTIR), and X-ray powder diffraction (XRD). The results obtained from characterisations indicate that the AgNPs have an almost spherical shape with a various size of 98.1 to $193 \mathrm{~nm}$. The synthesised nanoparticles exhibited outstanding antioxidant and antimicrobial activities.
\end{abstract}

Keywords: silver nanoparticles; nanoparticles; nanobiotechnology; Isochrysis.

\subsection{INTRODUCTION}

Nanotechnology is one of the most interesting studies in the current research world due to its vast applications in pharmaceuticals, medicine, and electronic fields. The synthesis of nanoparticles involves the particles size ranging from 1 to $100 \mathrm{~nm}$ (Naveena and Prakash, 2013). Electrochemical, sonochemical, and photochemical methods are some of the approaches being used to synthesise metallic nanoparticles. However, chemical method is not suitable due to the utilisation of toxic and hazardous chemicals, and involves difficult purification steps (Pak et al., 2016). Biological method combines the principles of biology with chemical and physical procedures to generate nanosized particles, which is termed as nanobiotechnology. Nanobiotechnology emphasises on economic alternatives and promotes nontoxic, ecofriendly, clean, and green synthesis method of nanoparticles. Biomolecules such as proteins, amino acids, 
carbohydrates, and sugars from microorganisms such as bacteria, fungi, and algae are used to biologically synthesise the metal nanoparticles (Chinnappan et al., 2015). Silver, gold, zinc, aluminium, titanium, palladium, iron, fullerenes, and copper are some of the metallic nanoparticles commonly synthesised via bionanotechnology procedures.

Silver nanoparticles (AgNPs) are one of the most important nanoparticles extensively studied in recent years due to its diverse beneficial properties and wide variety of applications in biological, chemical, and physical sciences. It is proved that silver nanoparticles possess unusual properties such as high antimicrobial activity, particles stability, and surface chemistry. Specific surface plasmon resonance (SPR) peak of silver nanoparticles falls in between 450 and $530 \mathrm{~nm}$. These different wavelengths are able to express different particle sizes, shapes, and surface properties of nanoparticles. Different physical formations lead to AgNPs to be widely used as antimicrobial and antifungal agents in healthcare, food, textile coatings, and electronic device industries. AgNPs which attached to the microbial cell surface cause structural changes of the cells and further lead to cell death by damaging the entire cell functions. Up to date, many commercial products incorporated with AgNPs were approved by FDA (USA), SIAA (Japan), KTR and FITI (Korea) (Kuppusamy et al., 2016).

Chemical methods such as sonochemical, polyols, matrix chemistry, sol gel, chemical reduction, and electrolysis are some of the proven metallic nanoparticles synthesising methods. Although these methods are efficient and fast, it also produces adverse effects to human and living organisms. Therefore, a sustainable method using biological medium as an alternative to conventional methods is essential to be developed. The synthesis of metal nanoparticles from readily available biological substances will allow the development of novel eco-friendly and cost effective procedures. Such eco-friendly metal nanoparticles synthesis particularly from marine algae were reported by Azizi et al. (2013), Mahdavi et al. (2013), Naveena and Prakash (2013), Patel et al. (2015), Singaravelu et al. (2007), and Venkatesan et al. (2014).

Microalgae is an autotrophic microorganism that consists of large taxa in the world. Isochrysis sp., a marine golden-brown flagellated microalga, is one of the prominent sources renowned for its potential pharmaceutical value through phyconanotechnology. Isochrysis sp. belongs to the phylum Haptophyta, class Coccolithopyhceae, subclass Prymnesiophycidae, order Isochrysidales, family Isochrysidaceae. Generally, with a small size of around 4-7 $\mu \mathrm{m}$, this microalga is able to grow very fast in a wide range of temperature, salinity, nutrients, and photoperiod. This fast growing microalga also rich with biopolymers containing glucose, galactose, mannose, xylose, arabinose, fucose, and rhamnose in various percentages. About $13 \%$ of dry matter of this microalga constituents are originated from carbohydrates derivatives. Usually, marine microalgae are prevalent in nutraceutical and pharmaceutical industries by providing high content of lipids, minerals, vitamins, secondary bioactive compounds such as polysaccharides, and proteins with potential medicinal property in fighting cancer, inflammation, allergy, and other metabolic diseases. These secondary metabolites can act as a functional group, reducing agents, and biological capped substances for all nanomaterials to play a role in antibiotic mechanisms against pathogens (Mohamed et al., 2012; Namvar et al., 2012; Zuercher et al., 2006). It was proven by the synthesis of cadmium nanomaterial using microalga Phaeodactylum sp. and biological reduction of gold nanoparticles by 
Rhizoclonium sp., Navicula sp., and Nitzschia sp. (Nayak et al., 2006; Scarano and Morelli, 2003).

In this study, brown microalga Isochrysis sp. was used for the synthesis of silver nanoparticles. Analytical techniques such as UV-visible spectroscopy, field emission scanning electron microscopy (FESEM), Fourier transform infrared spectroscopy (FTIR), and X-ray powder diffraction (XRD) were used to characterise the biologically synthesized AgNPs using Isochrysis sp. Furthermore, scavenging rate of 2,2-diphenyl1-picryl-hydrazyl (DPPH) as an indicator was used to study the antioxidant activity of AgNPs.

\section{Microalgae cultivation}

\subsection{METHODS}

Isochrysis sp. was cultivated aseptically at Bioprocess Laboratory, Faculty of Industrial Sciences and Technology, Universiti Malaysia Pahang. The F2 culture medium was used as microalgae nutrient support medium. The culture was grown in glass cylinders under indecent cool fluorescent light that was aided with clean sterile compressed aeration. Modified method from Naveena and Prakash (2013) was used to extract microalgal biomass cells. Microalgal cells were harvested by centrifugation for $10 \mathrm{~min}$ at 3,000 rpm. The collected cells were washed with sterile distilled water and then boiled with sterile distilled water. The extract was stored in a chiller until further use.

\section{Synthesis of AgNPs}

About $50 \mathrm{~mL}$ of Isochrysis sp. extract was mixed with $50 \mathrm{~mL}$ of freshly prepared $\mathrm{AgNO}_{3}(1 \mathrm{mM})$ solution and briskly stirred for $30 \mathrm{~min}$ at room temperature. Then, the mixture was allowed to stand at room temperature for $2 \mathrm{~h}$. The dark brown product was collected through centrifugation at $6,000 \mathrm{rpm}$ for $20 \mathrm{~min}$. The final product was dried at room temperature overnight and stored in an airtight container for further analysis (Azizi et al., 2013).

\section{Characterisation of AgNPs}

UV-visible spectroscopy, FESEM, FTIR, and XRD techniques were used to determine the size, shape, surface area, and dispersity of the nanoparticles produced. FESEM (JEOL JSM 7800F, USA) was used to observe the morphology of AgNPs. EDX analysis was conducted with the same FESEM instrument to determine the elemental composition of the synthesised AgNPs. For X-ray diffraction study, the synthesized silver nanoparticles were coated on XRD grid and $\mathrm{Cu}-\mathrm{Ka}$ radiation of $1.541 \AA$ at $30 \mathrm{kV}$ voltage and $15 \mathrm{~mA}$ current were used. FTIR spectra was recorded using PERKIN Elmer model at the resolution of $1 \mathrm{~cm}^{-1}$ in the range of 4000 to $400 \mathrm{~cm}^{-1}$. Thermogravimetric analysis (TGA) was monitored through Mettler-Toledo thermal analyser at a scanning rate of $10^{\circ} \mathrm{C} / \mathrm{min}$. 


\section{Antioxidant activity of AgNPs}

DPPH test was performed to estimate free radical scavenging activity as described by Phull et al., (2016). Different concentrations of silver nanoparticles were used to evaluate antioxidant activity of AgNPs. The silver nanoparticles were divided into aliquots of $2.5 \mathrm{~mL}$ and added into $1 \mathrm{~mL}$ of DPPH solution and made up to a final volume of $4 \mathrm{~mL}$. Once the solution was mixed properly, the absorbance value of the tested solution was read using a UV-visible spectrophotometer. The following formula was used to calculate the percentage of DPPH radical scavenging activity.

DPPH radical scavenging activity, $\%=\left(\frac{\text { Control OD-biosynthesized silver solution OD }}{\text { Control OD }}\right) \times 100$

\section{Antibacterial activity of AgNPs}

Antibacterial activity of AgNPs was performed by agar well diffusion method on selected human pathogens like Staphylococcus aureus and Escherichia coli. Biologically synthesised silver nanoparticles were conjugated with streptomycin before tested on pathogens at a ratio of 1:1. An aqueous solution of streptomycin was prepared as control. The strength of inhibition zone was measured after $24 \mathrm{~h}$ of incubation at 37 ${ }^{\circ} \mathrm{C}$ (Naveena and Prakash, 2013).

\subsection{RESULTS AND DISCUSSION}

In this study, the silver nanoparticles were synthesised biologically using microalga Isochrysis sp. The sizes, shapes and functional groups of the metal nanoparticles are reported. Antioxidant and antimicrobial activities of the synthesised silver nanoparticles are ascertained.

Figure (1) shows the colour change of silver nanoparticles from pale yellow to brown. The colour of the solution changed to brown within $2 \mathrm{~h}$. There are a few reports on the synthesis of silver and gold nanoparticles using macroalgae, such as Sargassum muticum (Azizi et al., 2013) and various types of green microalgae (Patel et al., 2015) for silver nanoparticles, and Sargassum wightii (Singaravelu et al., 2007), Gracilaria corticata (Naveena and Prakash, 2013); Turbinaria conoides, and Sargassum tenerrimum (Ramakrishna et al., 2016) for gold nanoparticles.

The UV-visible spectroscopy at light wavelengths of $200-800 \mathrm{~nm}$ is a commonly used technique to characterise various metal nanoparticles in the size range of $2-100 \mathrm{~nm}$ (Manivasagan and Kim, 2015). In this study, AgNPs solutions were subjected to different UV-spectrum wavelengths ranging from 300 to $700 \mathrm{~nm}$ and showed maximum absorption peak at $414 \mathrm{~nm}$ (Figure 2). The UV-visible absorption spectrum is a strong evidence that the metal nanoparticles formed in different sizes and shapes in the medium. Resonant peak occurs at various wavelengths for different types of nanoparticles mixture. This factor is termed as surface plasmon resonance (SPR). According to SPR theory, the maximum resonance wavelength is absorbed at resonant wavelength. Molecular stability, shape, and size of particles in the medium or upon inner particles distance and its surrounding media are the factors influencing SPR patterns of metallic nanoparticles. Various previous studies suggested a usual silver nanoparticles SPR pattern at wavelength in the range of 400-480 $\mathrm{nm}$ (Haghighi Pak et al., 2016; Manivasagan and Kim, 2015). In this UV-visible spectrum analysis, broad SPR peak was observed at $414 \mathrm{~nm}$. This result confirmed the formation of AgNPs from 
Isochrysis sp. extract. Past researches suggested that spherical shaped nanoparticles (Figure 3) was attributed to SPR peak between 410 and $450 \mathrm{~nm}$ (Azizi et al., 2013; Devi and Bhimba, 2012; Jyoti et al., 2016).

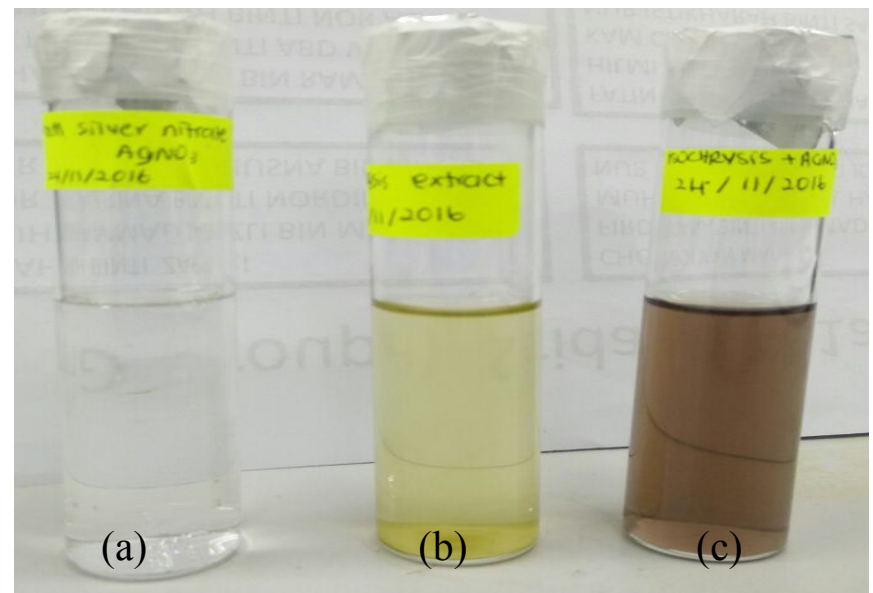

Figure 1: (a) Silver nitrate $\left(\mathrm{AgNO}_{3}\right)$ solution $(1 \mathrm{mM})$, (b) Microalga extract, (c) Brown colour formation after the reaction of $1 \mathrm{mM}$ of $\mathrm{AgNO}_{3}$ with microalga extract.

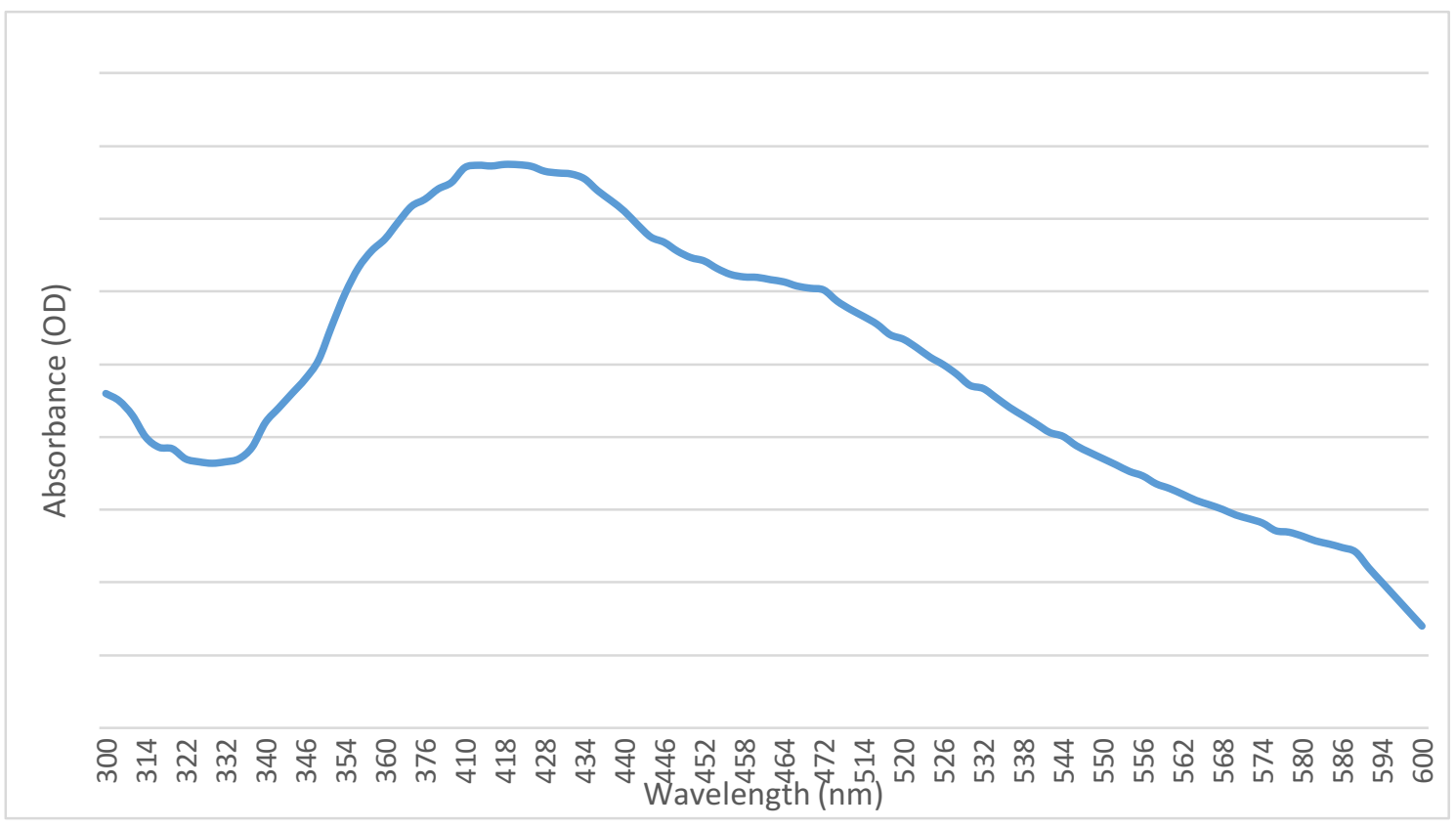

Figure 2: UV-visible absorbance curve of AgNPs synthesised using Isochrysis sp. 
The size and morphology of the synthesised AgNPs were determined through FESEM images, as shown in Figure 3. The morphology of AgNPs is almost spherical with an average size ranges from 98.1 to $193 \mathrm{~nm}$. Through FESEM images, it was confirmed that the synthesised nanoparticles were monodispersed in terms of particle size. Moreover, Pak et al., (2016) revealed that not indirect contact of monodispersed of AgNPs are the indication of stable nanoparticles through capping agents. The capping ligand could be of a carbonyl group, an aromatic compound, alkanes, or amines (Vivek et al., 2011). Such bio-capped molecules will help to prevent agglomeration of NPs and at the same time will enhance antimicrobial activity. In this study, aggregated AgNPs with indirect contact of AgNPs was observed in Figure 4.
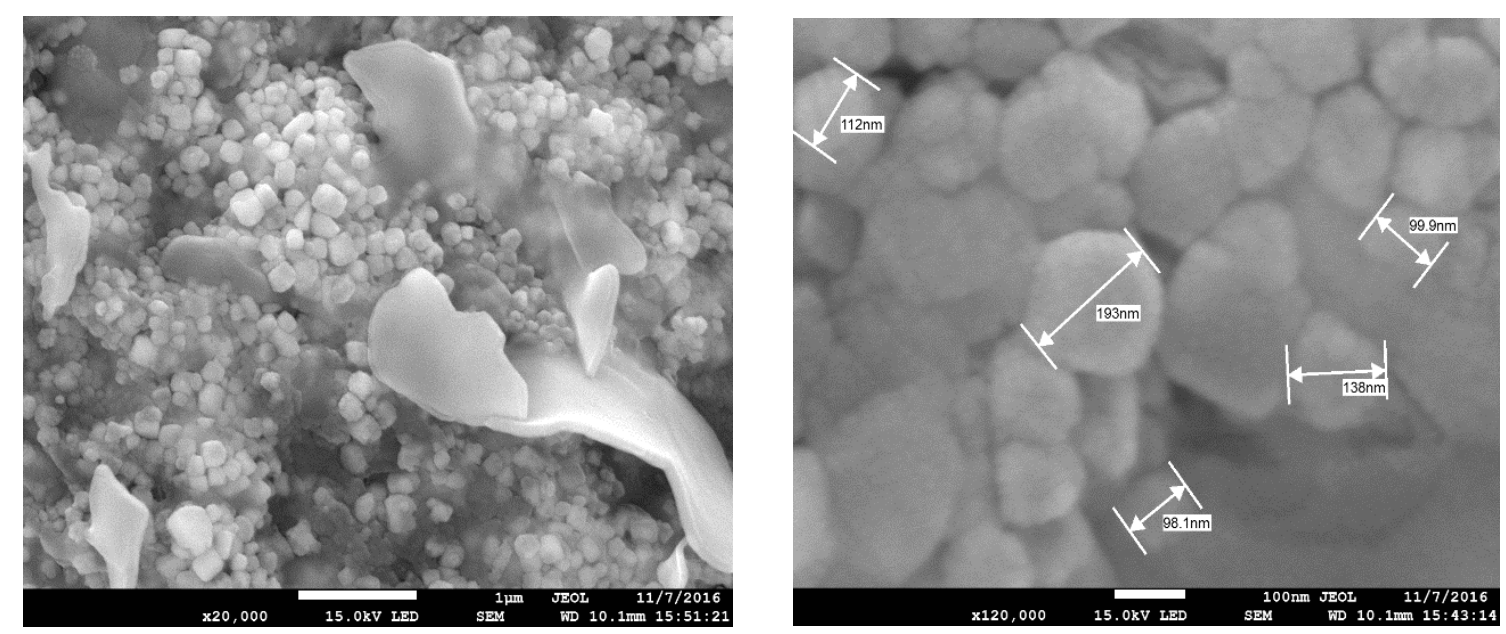

Figure 3: Field emission scanning electron microscope (FESEM) images of silver nanoparticles $\left(\mathrm{AgNO}_{3}\right)$ synthesised through Isochrysis sp.
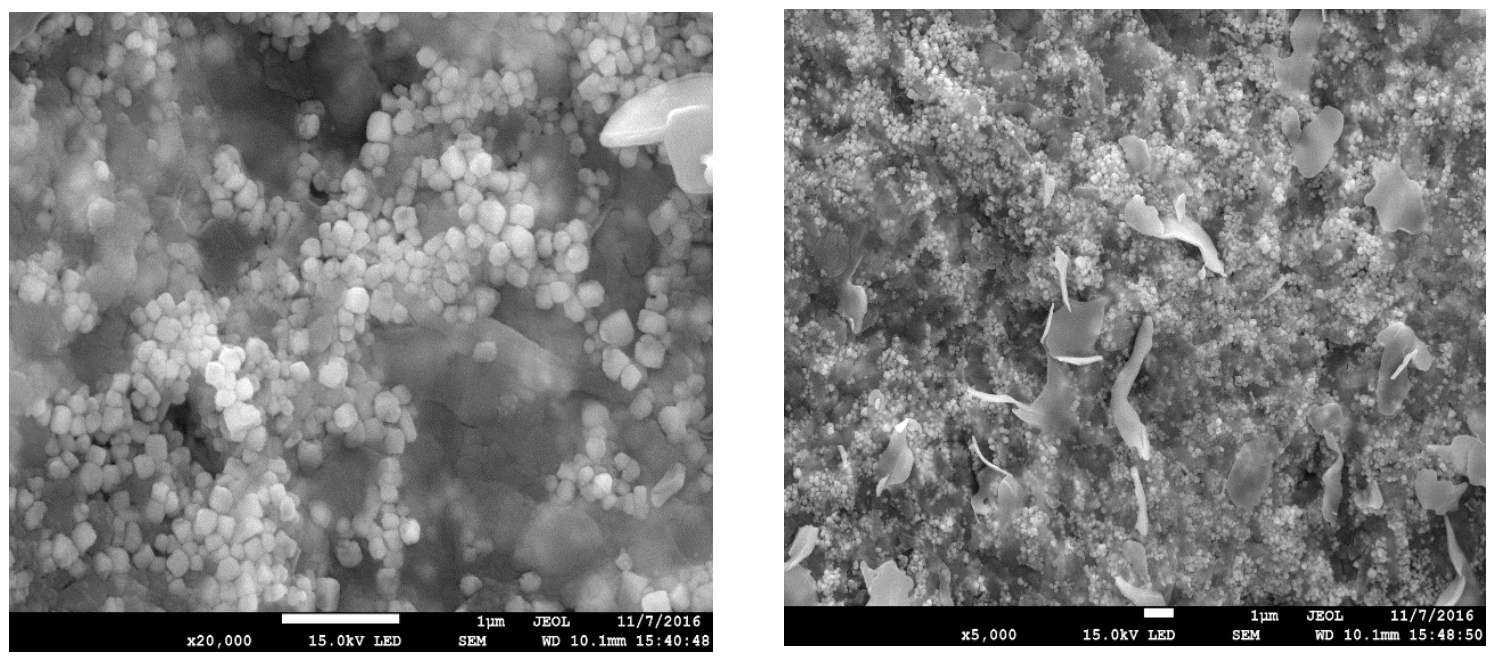

Figure 4: Monodispersed AgNPs with indirect contact of nanoparticles. 
Figure 5 shows EDX spectra of the synthesised AgNPs. The spectra confirmed that Ag was expressed where the silver composition was nearly $43 \%$ in the sample. The strong signal energy peaks of silver atoms were observed in the range between 3 and $4 \mathrm{keV}$. Weaker signals are observed for $\mathrm{C}, \mathrm{O}, \mathrm{N}, \mathrm{Mg}, \mathrm{Si}$, and $\mathrm{Cl}$ (Table 1). Carbon, oxygen, nitrogen, magnesium, silicon, and chlorine elements that shown in the EDX spectra which is dominant compounds in the secondary metabolites of the plant extract (Figure $5 \mathrm{a}, \mathrm{b})$. Similar compounds were observed by Mahdavi et al., (2013) and Ravichandran et al., (2015).

Through X-ray diffraction pattern (XRD), four peaks were detected at $22.3^{\circ}, 28.3^{\circ}$, $32.9^{\circ}$, and $46.6^{\circ}$ which are assigned to (111), (220), (122), and (231), respectively. This observation revealed that AgNPs that were synthesised from Isochrysis sp. consisted of pure crystalline Ag. Similarly, silver nanostructure which was obtained from Gelidiella acerosa extract was confirmed by the characteristic peak observed at $2 \theta=28.09^{\circ}$ marked with (220). Thus, Bragg reflections corresponding to the (220) sets of lattice planes based on the face-centred crystal structure of silver were predicted (Vivek et al., 2011). In addition, similar results were recorded by Gnanajobitha et al., (2013) and Jyoti et al. (2015) with facets of face-centred cubic crystal structure of silver nanoparticles.

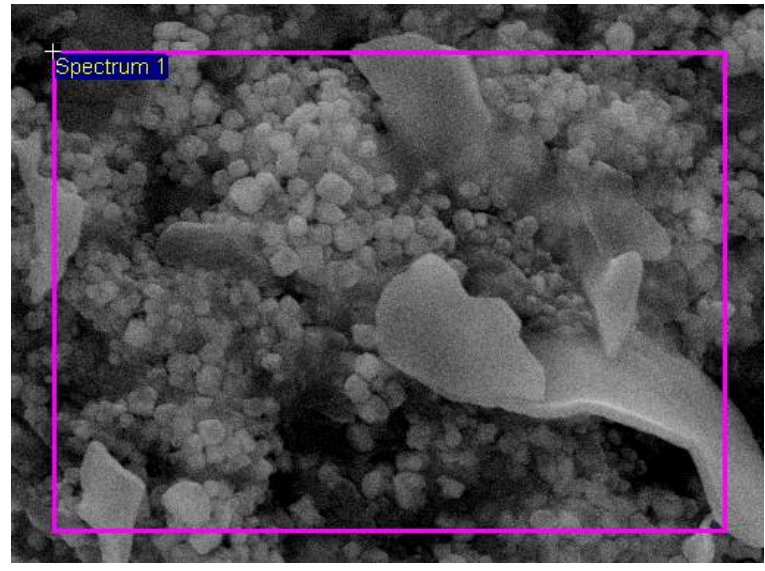

(a)

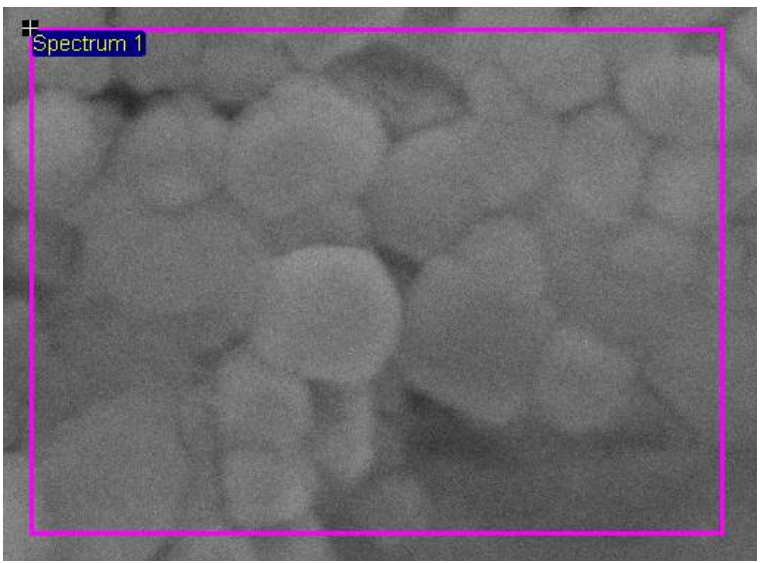

(c)
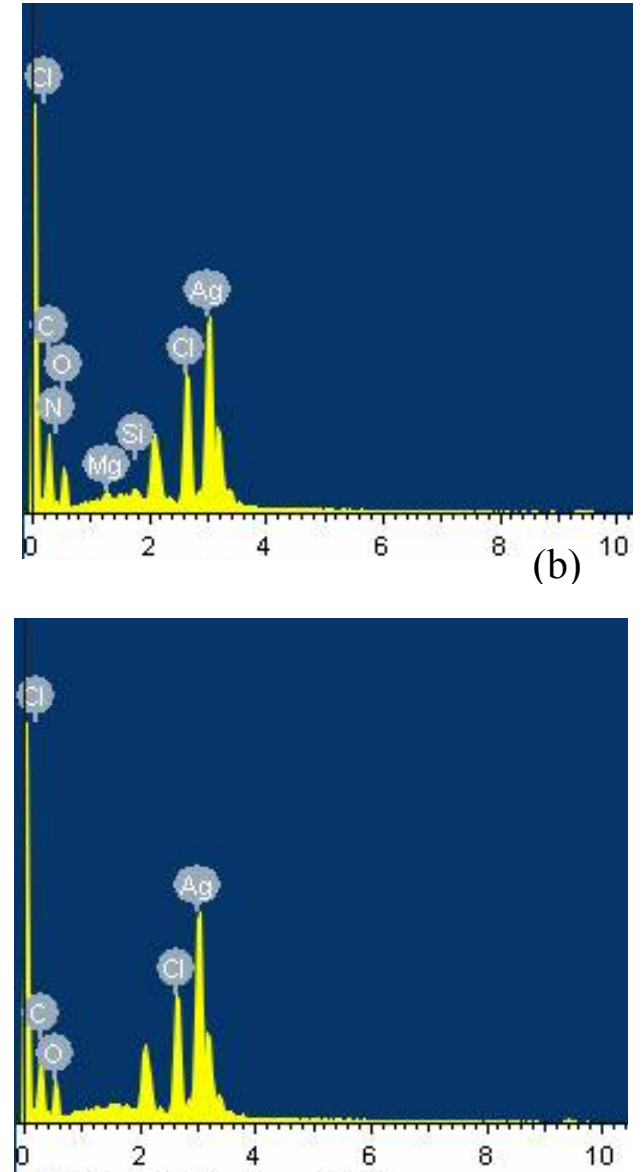

(d)

Figure 5: EDX results of (a) and (b) nanoparticles inclusive dead microalgae cells and (c) and (d) nanoparticles only. 
Table 1: The elements in AgNPs determined by EDX

\begin{tabular}{ccc}
\hline Element & Weight \% & Atomic \% \\
\hline $\mathrm{C}$ & 21.30 & 43.20 \\
$\mathrm{~N}$ & 8.56 & 14.89 \\
$\mathrm{O}$ & 15.92 & 24.25 \\
$\mathrm{Mg}$ & 0.47 & 0.47 \\
$\mathrm{Si}$ & 0.61 & 0.53 \\
$\mathrm{Cl}$ & 10.10 & 6.94 \\
$\mathrm{Ag}$ & 43.05 & 9.72 \\
Total & 100.00 & 100.00 \\
\hline
\end{tabular}

Fourier transform infrared spectroscopy (FTIR) measurement was carried out to identify the biomolecules that bound on the silver surface (Xie et al., 2007). Figure (6) shows the FTIR spectrum of the biosynthesised silver nanoparticles. Absorbance bands were seen at $602,1053,1535,1637,2848,2917$, and $3448 \mathrm{~cm}^{-1}$. Strong absorption band at $602 \mathrm{~cm}^{-1}$ could be a C-H alkene band. Strong peak observed at $1053 \mathrm{~cm}^{-1} \mathrm{C}-\mathrm{O}$ could be bending esters and the peak at $1637 \mathrm{~cm}^{-1}$ is attributed to $\mathrm{C}=\mathrm{O}$ stretching carbonyls. The high absorbance bond was observed at $3448 \mathrm{~cm}^{-1}$ which indicates deforming vibration of $\mathrm{O}-\mathrm{H}$ strong bond stretching phenols and $\mathrm{N}-\mathrm{H}$ stretching primary and secondary amines and amides. Also, $\mathrm{C}-\mathrm{H}$ stretching aldehyde, $\mathrm{C}-\mathrm{H}$ stretching alkene, and aromatics $\mathrm{N}-\mathrm{H}$ amine were observed at 2848,2917 , and $1535 \mathrm{~cm}^{-1}$, respectively. The presence of amines, amides, phenols, and carboxyl in the synthesised AgNPs are shown in FTIR analysis results. It is recommended that during the reduction with silver ion, the $\mathrm{C}-\mathrm{OH}$ alcohol group is converted to the carbonyl group $\mathrm{C}=\mathrm{O}$. Carbonyl group plays a role as a stabilizing agent for $\mathrm{Ag}$ nanoparticles. The following equation $\mathrm{Ag}^{+}+\mathrm{R}-\mathrm{OH} \rightarrow$ $\mathrm{R}=\mathrm{O}+\mathrm{Ag}+\mathrm{H}^{+}$explains the reaction between the microalgae extract and silver ions (Meng, 2015). Meanwhile, earlier reports indicated that the stabilisation of silver nanoparticles is also possible when free amine groups or cysteine residues in the proteins bind with nanoparticles via electrostatic attraction of negatively charged carboxylate groups (Sanghi and Verma, 2009).

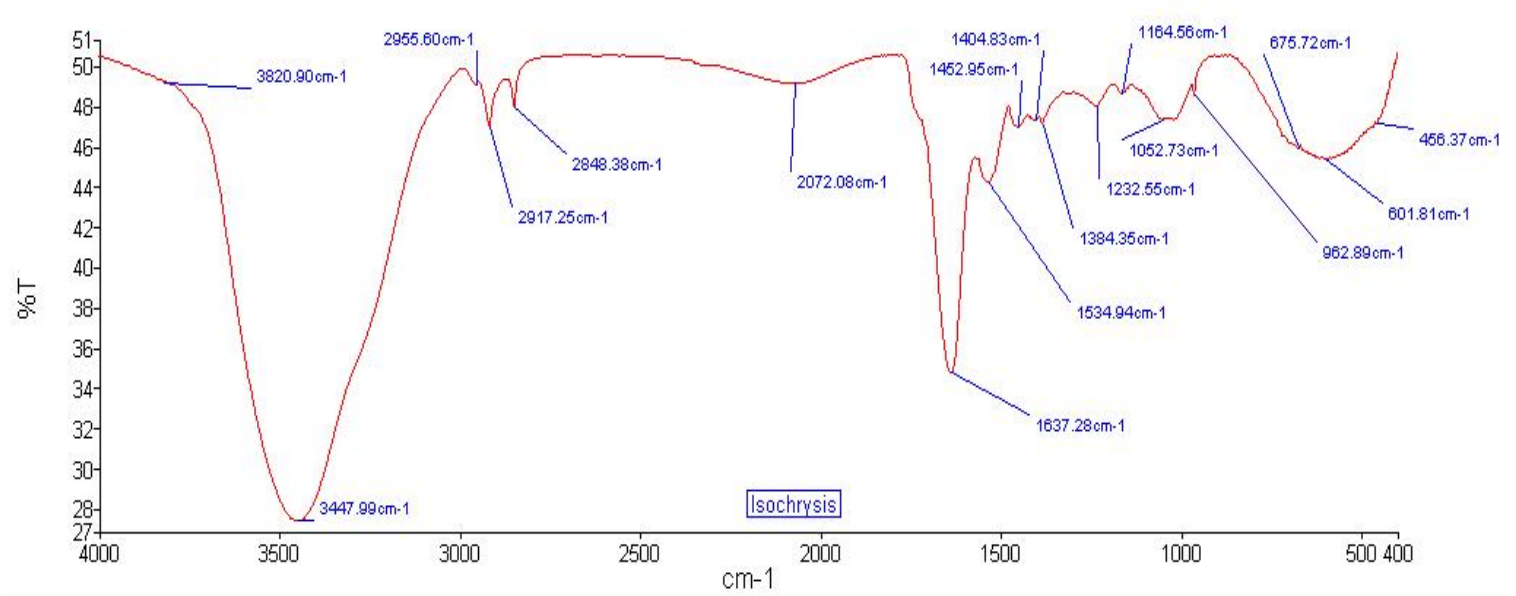

Figure 6: FTIR spectra of the synthesised AgNPs using Isochrysis sp. extract 
TGA curve of the silver nanoparticles synthesised using Isochrysis sp. is shown in Figure 7. The samples was heated from 50 to $700{ }^{\circ} \mathrm{C}$ which was in between the boiling point of the solvent and the degradation temperature of polymer (Alahmad et al., 2013). In this study, two main weight losses were observed in the TGA graph. The first weight loss was observed at $55^{\circ} \mathrm{C}$. Water molecules losses believe for the first weight loss. The second weight loss was recorded at the temperature between 290 and $320{ }^{\circ} \mathrm{C}$. It can be attributed to the evaporation of organic components occurred at this stage. Moreover, complete thermal decomposition and crystallisation of the sample happened concurrently. Similar trend was observed by Khan et $a$. (2011) between 200 and $300{ }^{\circ} \mathrm{C}$.

Radical scavenging activity of a compound generally is tested via DPPH test. DPPH test is an easy and rapid method to analyse antioxidant activity. The biosynthesised silver nanoparticles were tested for the potential free radical scavenging activity. The effective free radical scavenging potential of Isochrysis sp. derived silver nanoparticles and Isochrysis sp. extract were $58.8 \%$ and $28.4 \%$, respectively. The presence of nanoparticles improved the scavenging activity compared to normal microalga extract. Saranya et al. (2014) indicated that a maximum $34.18 \%$ of scavenging activity was found in methanol extract of Isochrysis galbana.

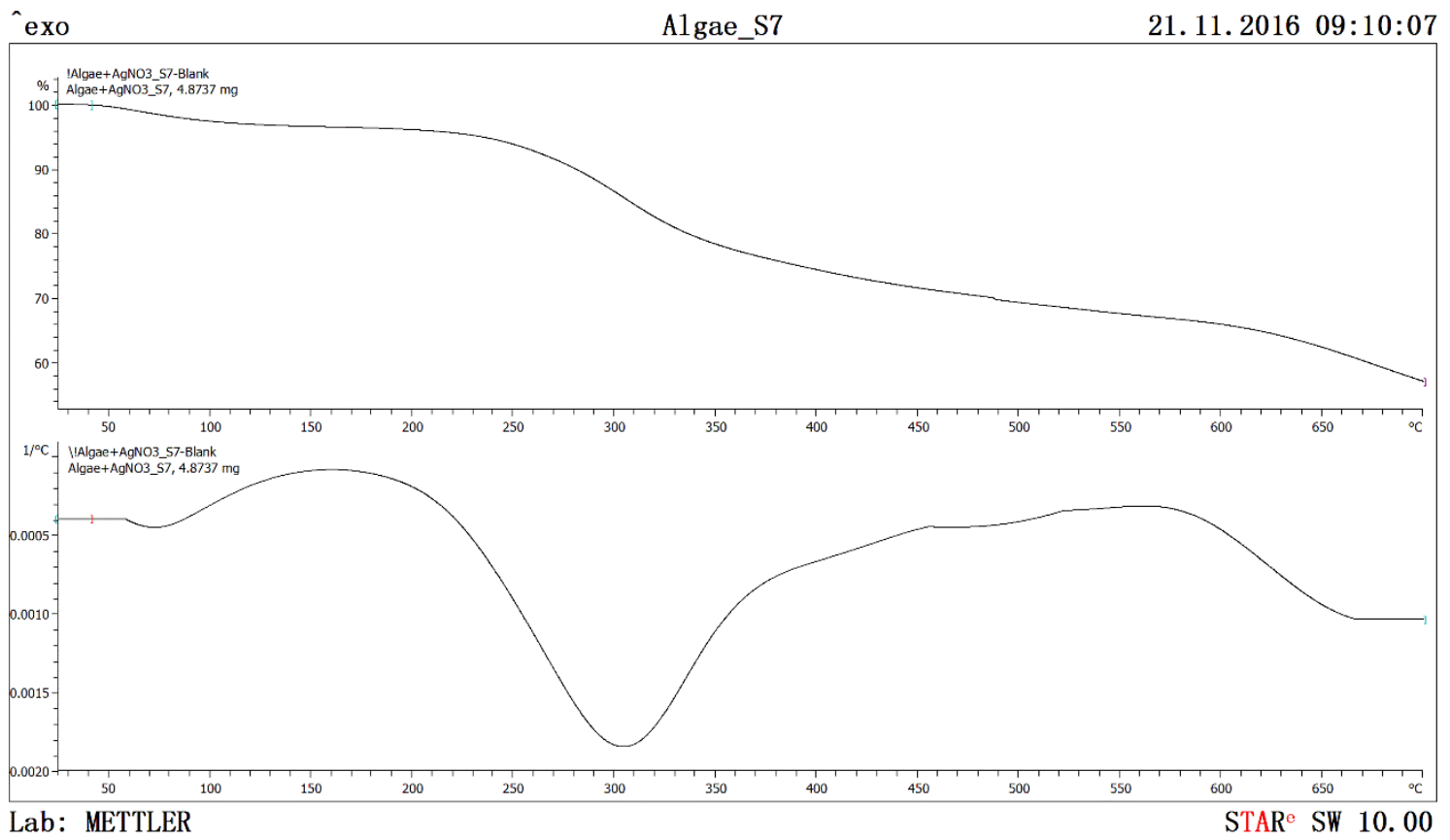

Figure 7: TGA thermogram of Isochrysis sp. synthesised silver nanoparticles

Silver nanoparticles are well known for their antibacterial effects. Table (2) shows the antibacterial activity of AgNPs against $S$. aureus and E. coli. As predicted, maximum zones of inhibition were observed in the antibiotic conjugated silver nanoparticles. A maximum antimicrobial activity was observed against $E$. coli with $15 \mathrm{~mm}$ inhibition zone. About $10 \mathrm{~mm}$ of inhibition zone was observed against $S$. aureus. Although only two types of bacterial strain used in this study, the synthesised AgNPs were able to be used against gram-positive and gram-negative bacteria. Whereas, Sriram and Pandidurai (2014) demonstrated that AgNPs synthesised from Psidium guajava was able to inhibit the growth of $S$. aureus and E. coli. Gnanajobitha et al. (2013) verified that 
biosynthesised AgNPs from Vitis vinifera was able to inhibit the growth of Bacillus subtilis and Klebsiella planticola.

Moreover, Pak et al. (2016) validated that biologically synthesised AgNPs exhibited greater antimicrobial activity when compared with chemically synthesised AgNPs. The small size of biosynthesised nanoparticles and the presence of bioactive compound capping may contribute for enhanced activity of bio-nanoparticles. AgNPs were able to inhibit the growth of Bacillus subtilis and Klebsiella planticola (Gnanajobitha et al., 2013

Table 2: Antibacterial activity of biosynthesised silver nanoparticles

\begin{tabular}{lcc}
\hline \multicolumn{1}{c}{ Bacterial Strain } & \multicolumn{2}{c}{ Inhibition zones in mm } \\
\cline { 2 - 3 } & Streptomycin & Streptomycin + AgNPs \\
\hline Staphylococcus aureus & $6.0 \pm 0.5$ & $10.5 \pm 0.5$ \\
Escherichia coli & $8.0 \pm 0.5$ & $15.5 \pm 0.5$ \\
\hline
\end{tabular}

\subsection{CONCLUSION}

In the present study, microalga Isochrysis sp. was used to synthesise AgNPs at room temperature. The preparation method was a simple and environmentally friendly approach. Additionally, the biosynthesised AgNPs can be used in medical and other applications. The current study demonstrated that Isochrysis sp. reduced silver nanoparticles were capable of inhibiting bacterial growth and showed antioxidant potential as well. On top of that, studies on Isochrysis sp. synthesised AgNPs will give a new insight for a new range of antibacterial and antioxidant agents. Further research is required to identify the potential of microalga derived AgNPs and compounds responsible to synthesise AgNPs from microalga. Therefore, optimisation of biosynthesis of AgNPs can be highlighted in future.

\section{ACKNOWLEDGEMENTS}

The author gratitude goes to the staff of the Central Laboratory, UMP and Faculty of Industrial Sciences \& Technology (FIST) for their technical aid. The project is funded by internal grant of Universiti Malaysia Pahang.

\section{REFERENCES}

Alahmad, A., Eleoui, M., Falah, A. and Alghoraibi, I. (2013). Preparation of colloidal structural characterization silver nanoparticles and structural characterization. Physical Sciences Research International, 1(4), 89-96.

Azizi, S., Namvar, F., Mahdavi, M., Ahmad, M. and Mohamad, R. (2013). Biosynthesis of silver nanoparticles using brown marine macroalga, Sargassum muticum aqueous extract. Materials, 6(12), 5942-5950.

Chinnappan, R. S., Kandasamy, K., and Sekar, A. (2015). A review on marine based nanoparticles and their potential applications. African Journal of Biotechnology, 14(18), 1525-1532.

Devi, J. S. and Bhimba, B. V. (2012). Silver nanoparticles: Antibacterial activity against wound isolates \& in vitro cytotoxic activity on human Caucasian colon adenocarcinoma. Asian Pacific Journal of Tropical Disease, 2, S87-S93.

Gnanajobitha, G., Paulkumar, K., Vanaja, M., Rajeshkumar, S., Malarkodi, C., Annadurai, G. and Kannan, C. (2013). Fruit-mediated synthesis of silver nanoparticles using Vitis vinifera and evaluation of their antimicrobial efficacy. Journal of Nanostructure in Chemistry, 3(1), 67.

Haghighi Pak, Z., Abbaspour, H., Karimi, N. and Fattahi, A. (2016). Eco-friendly synthesis and antimicrobial activity of silver nanoparticles using Dracocephalum moldavica seed extract. Applied Sciences, 6(3), 69. 
Jyoti, K., Baunthiyal, M. and Singh, A. (2016). Characterization of silver nanoparticles synthesized using Urtica dioica Linn. leaves and their synergistic effects with antibiotics. Journal of Radiation Research and Applied Sciences, 9(3), 217-227.

Mahdavi, M., Namvar, F., Ahmad, M. Bin. and Mohamad, R. (2013). Green biosynthesis and characterization of magnetic iron oxide $\left(\mathrm{Fe}_{3} \mathrm{O}_{4}\right)$ nanoparticles using seaweed (Sargassum muticum) aqueous extract. Molecules (Basel, Switzerland), 18(5), 5954-64.

Majeed Khan, M. A., Kumar, S., Ahamed, M., Alrokayan, S. A. and Alsalhi, M. S. (2011). Structural and thermal studies of silver nanoparticles and electrical transport study of their thin films. Nanoscale Research Letters, 6(1), 434.

Manivasagan, P. and Kim, S.-K. (2015). Biosynthesis of nanoparticles using marine algae: A review. In Kim, S.-K. and Chojnacka, K. (Ed.) Marine Algae Extracts (pp. 295-304). Weinheim, Germany: Wiley-VCH Verlag GmbH \& Co. KGaA.

Meng, Y. (2015). A sustainable approach to fabricating Ag nanoparticles/PVA hybrid nanofiber and its catalytic activity. Nanomaterials, 5(2), 1124-1135.

Mohamed, S., Hashim, S. N. and Rahman, H. A. (2012). Seaweeds: A sustainable functional food for complementary and alternative therapy. Trends in Food Science and Technology, 23(2), 83-96.

Namvar, F., Mohamed, S., Fard, S. G., Behravan, J., Mustapha, N. M., Alitheen, N. B. M. and Othman, F. (2012). Polyphenol-rich seaweed (Eucheuma cottonii) extract suppresses breast tumour via hormone modulation and apoptosis induction. Food Chemistry, 130(2), 376-382.

Naveena, B. E. and Prakash, S. (2013). Biological synthesis of gold nanoparticles using marine algae Gracilaria corticata and its aplplication as a potent antimicrobial and antioxidant agent. Asian Journal of Pharmaceutical and Clinical Research, 6(2), 4-7.

Nayak, D., Nag, M., Banerjee, S., Pal, R., Laskar, S. and Lahiri, S. (2006). Preconcentration of 198Au in a green alga, Rhizoclonium. Journal of Radioanalytical and Nuclear Chemistry, 268(2), 337-340.

Kuppusamy, P., Ichwan, S. J., Al-Zikri, P. N., Suriyah, W. H., Soundharrajan, I., Govindan, N., Maniam, G. P. and Yusoff, M. M. (2016). In vitro anticancer activity of au, ag nanoparticles synthesized using Commelina nudiflora $l$. aqueous extract against HCT-116 colon cancer cells. Biological Trace Element Research, 173(2), 297-305.

Patel, V., Berthold, D., Puranik, P. and Gantar, M. (2015). Screening of cyanobacteria and microalgae for their ability to synthesize silver nanoparticles with antibacterial activity. Biotechnology Reports, 5(1), 112-119.

Phull, A.-R., Abbas, Q., Ali, A., Raza, H., kim, S. J., Zia, M. and Haq, I. (2016). Antioxidant, cytotoxic and antimicrobial activities of green synthesized silver nanoparticles from crude extract of Bergenia ciliata. Future Journal of Pharmaceutical Sciences, 2(1), 31-36.

Ramakrishna, M., Rajesh Babu, D., Gengan, R. M., Chandra, S. and Nageswara Rao, G. (2016). Green synthesis of gold nanoparticles using marine algae and evaluation of their catalytic activity. Journal of Nanostructure in Chemistry, 6(1), 1-13.

Ravichandran, S., Paluri, V., Kumar, G., Loganathan, K. and Kokati Venkata, B. R. (2016). A novel approach for the biosynthesis of silver oxide nanoparticles using aqueous leaf extract of Callistemon lanceolatus (Myrtaceae) and their therapeutic potential. Journal of Experimental Nanoscience, 11(6), 445-458.

Sanghi, R. and Verma, P. (2009). Biomimetic synthesis and characterisation of protein capped silver nanoparticles. Bioresource Technology, 100(1), 501-504.

Saranya, C., Hemalatha, A., Parthiban, C. and Anantharaman, P. (2014). Evaluation of antioxidant properties, total phenolic and carotenoid content of Chaetoceros calcitrans, Chlorella salina and Isochrysis galbana. International Journal of Current Microbiology and Applied Sciences, 3(8), $365-377$.

Scarano, G. and Morelli, E. (2003). Properties of phytochelatin-coated CdS nanocrystallites formed in a marine phytoplanktonic alga (Phaeodactylum tricornutum, Bohlin) in response to Cd. Plant Science, 165(4), 803-810.

Singaravelu, G., Arockiamary, J. S., Kumar, V. G. and Govindaraju, K. (2007). A novel extracellular synthesis of monodisperse gold nanoparticles using marine alga, Sargassum wightii Greville. Colloids and Surfaces B: Biointerfaces, 57, 97-101.

Sriram, T. and Pandidurai, V. (2014). Synthesis of silver nanoparticles from leaf extract of Psidium guajava and its antibacterial activity against pathogens. International Journal of Current Microbiology and Applied Sciences, 3(3), 146-152.

Venkatesan, J., Manivasagan, P., Kim, S. K., Kirthi, A. V., Marimuthu, S. and Rahuman, A. A. (2014). Marine algae-mediated synthesis of gold nanoparticles using a novel Ecklonia cava. Bioprocess and Biosystems Engineering, 37(8), 1591-1597. 
Vivek, M., Kumar, P. S., Steffi, S. and Sudha, S. (2011). Biogenic silver nanoparticles by Gelidiella acerosa extract and their antifungal effects. Avicenna Journal of Medical Biotechnology, 3(3), 143148.

Xie, J., Lee, J. Y., Wang, D. I. C. and Ting, Y. P. (2007). Identification of active biomolecules in the highyield synthesis of single-crystalline gold nanoplates in algal solutions. Small, 3(4), 672-682.

Zuercher, A. W., Fritsché, R., Corthésy, B. and Mercenier, A. (2006). Food products and allergy development, prevention and treatment. Current Opinion in Biotechnology, 17(2), 198-203. 